\title{
ArcheoSciences
}

Revue d'archéométrie

$37 \mid 2013$

Varia

\section{Sites à stratification complexe et modélisation tridimensionnelle : vers une nouvelle approche des sépultures multiples}

Stratified and Complex Sites, Three-Dimensional Modeling: Towards a New

Approach to Multiple Burials

Géraldine Sachau-Carcel, Dominique Castex et Robert Vergnieux

\section{OpenEdition \\ Journals}

Édition électronique

URL : https://journals.openedition.org/archeosciences/4000

DOI : 10.4000/archeosciences.4000

ISBN : 978-2-7535-2755-3

ISSN : 2104-3728

Éditeur

Presses universitaires de Rennes

Édition imprimée

Date de publication : 17 avril 2013

Pagination : 89-104

ISBN : 978-2-7535-2757-7

ISSN : $1960-1360$

Référence électronique

Géraldine Sachau-Carcel, Dominique Castex et Robert Vergnieux, " Sites à stratification complexe et modélisation tridimensionnelle : vers une nouvelle approche des sépultures multiples »,

ArcheoSciences [En ligne], 37 | 2013, mis en ligne le 17 avril 2015, consulté le 28 janvier 2022. URL

http://journals.openedition.org/archeosciences/4000; DOI : https://doi.org/10.4000/archeosciences. 4000 


\title{
Sites à stratification complexe et modélisation tridimensionnelle: vers une nouvelle approche des sépultures multiples
}

\author{
Stratified and Complex Sites, Three-Dimensional Modeling: \\ Towards a New Approach to Multiple Burials
}

\author{
Géraldine Sachau-Carcel*, Dominique Castex** et Robert Vergnieux***
}

Résumé: En 2003, est mis au jour un secteur jusqu'alors inexploré de la catacombe des Saints Pierre-et-Marcellin, au sud-est de Rome. Il se présente sous la forme de plusieurs salles remplies d'un nombre considérable de squelettes évoquant des dépôts multiples, un mode d'inhumation jusqu'alors inconnu au sein de cette catacombe pour laquelle l'inhumation individuelle reste dominante pour la période concernée ( $\mathrm{I}^{\mathrm{er}} \mathrm{s}$. apr. J.-C. milieu $\mathrm{III}^{\mathrm{e}}$ s. apr. J.-C.). La complexité de l'organisation interne de ces tombes à dépôts multiples a motivé l'emploi de nouvelles techniques de représentations telles que la modélisation tridimensionnelle. Pour chaque tombe multiple, l'utilisation de la scène numérique a permis de mener précisément différentes analyses telles que la compréhension de la gestion des corps et de la logique interne des dépôts, contribuant ainsi à enrichir la réflexion archéologique sur ces ensembles.

\begin{abstract}
In 2003, a part of Saints Peter and Marcellinus catacombs -in south-east of Rome-, unexplored and unique in their organisation is discovery. Several graves (dated the 1st-3rd c. A.D.) contain many buried people, most of whom appear to have been inhumed. This method of deposit is hitherto unknown in this catacomb for the period concerned the individual burial dominant. The internal organisation is complex and required the use of new techniques such as representations of three-dimensional modelling. The studies of this mass grave are based on the comprehension of relationships between bodies and the taphonomic evolution of the deposits for each grave. The use of numerical scene have enabled to conduct precisely different studies and infirm or confirm the archaeological presupposed, to enrich the reflexion.
\end{abstract}

Mots-clés: Rome, catacombe, sépultures multiples, modélisation tridimensionnelle, archéologie de la mort.

Keyword: Rome, catacomb, multiple burials, three-dimensional modeling, death archaeology.

\footnotetext{
* Doctorante université Bordeaux 3 - Bordeaux 1, UMR 5607 Ausonius, 8 esplanade des Antilles 33607 Pessac cedex, auteur de correspondance. (g.sachau@wanadoo.fr)

**Université Bordeaux 1,UMR 5199, PACEA-A3P, B8, avenue des facultés 33405 Talence cedex. (d.castex@.u-bordeaux1.fr)

***Université Bordeaux 3, UMR 5607, Ausonius, 8 esplanade des Antilles 33607 Pessac cedex. (vergnieux@u-bordeaux3.fr)
} 


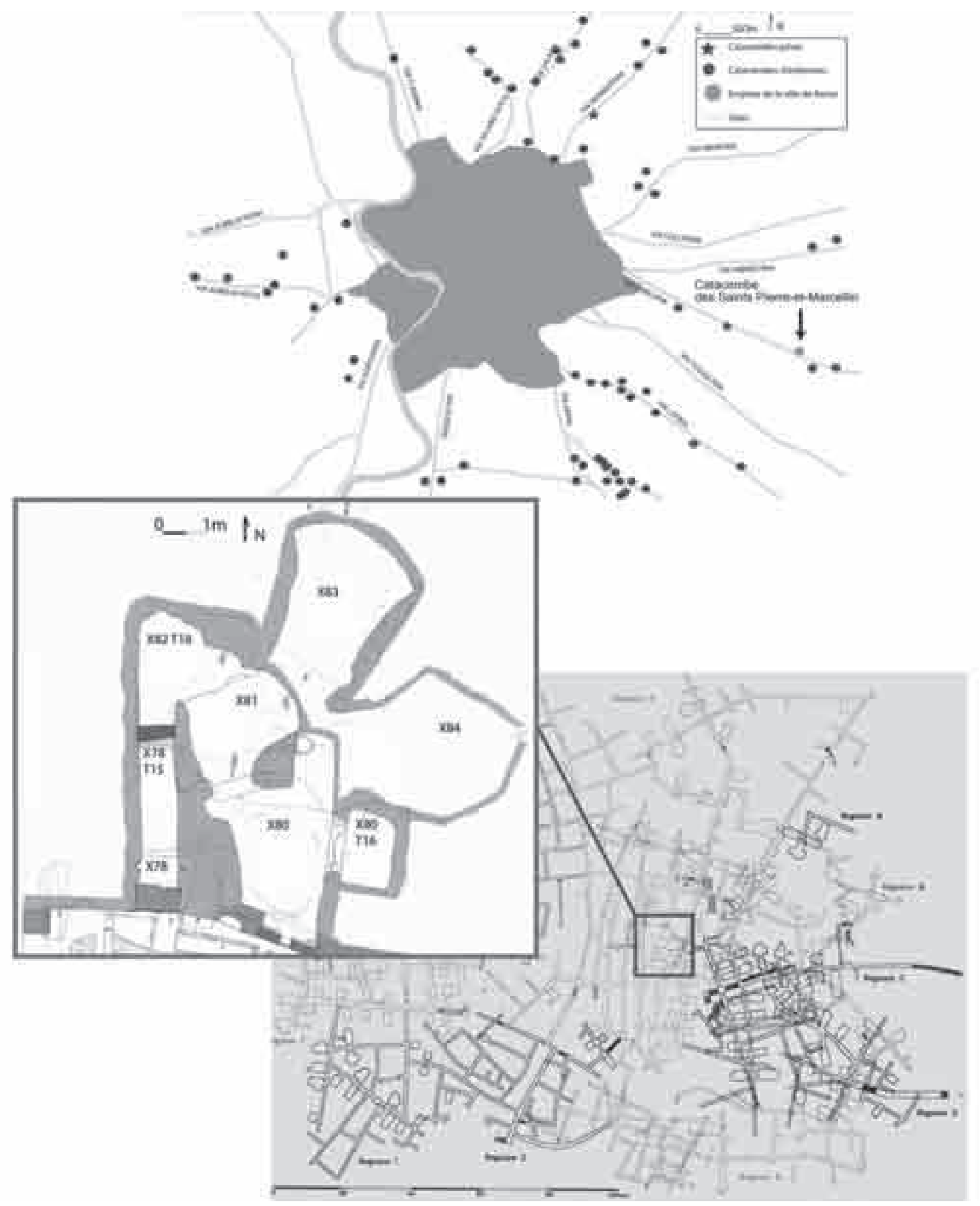

Figure 1: Localisation de la catacombe des Saints Pierre-et-Marcellin et plan des catacombes avec le secteur central (encadré). Figure 1: Localisation of Saints Peter and Marcellinus catacomb and map of the central sector (box). 


\section{INTRODUCTION}

Le développement de nouveaux outils informatiques nous amène à renouveler les techniques d'enregistrement et d'analyse tant dans le domaine archéologique que dans le domaine anthropologique. Les sépultures à dépôts multiples, en raison de leur complexité, représentent un champ d'expérimentation pour les scènes numériques tridimensionnelles. La récente découverte d'un ensemble de tombes multiples, au sein d'un secteur de la catacombe des Saints Pierre-et-Marcellin à Rome (Italie), nous a permis de tester l'utilisation de nouvelles techniques d'étude de ces ensembles funéraires à la stratification complexe. L'objectif de notre recherche, menée dans le cadre d'un doctorat ${ }^{1}$, a consisté en l'étude précise de la gestion des dépôts afin de mettre en évidence les relations de simultanéité entre les corps, premier argument pour une interprétation de crises de mortalité, mais également entre les différents niveaux de dépôts identifiés lors de la fouille. Préalablement à cette étude, il a été nécessaire d'élaborer et de tester une méthodologie pour l'acquisition des données permettant de restituer les individus et leur traitement funéraire, les différentes tombes, et l'espace funéraire dans sa globalité.

\section{Présentation}

La catacombe des Saints Pierre-et-Marcellin, une des soixante catacombes chrétiennes de la ville de Rome, est située au sud-est de la ville, le long de la via Casilina (ancienne voie Labicana), à proximité du mausolée d'Hélène. Le secteur central contenant un ensemble de sépultures multiples a été mis au jour en 2003 (fig. 1). Après un diagnostic mené par une archéologue de la Commission Pontificale d'Archéologie Sacrée, la responsabilité de la fouille et de l'étude de ce secteur de la catacombe a été confiée à une équipe française de l'UMR 5199 PACEA-A3P associée à des archéologues de l'Inrap (Castex et al., 2007: 274-282, Giuliani et al., 2007: 83-124).

Les pratiques funéraires observées au sein de ces ensembles funéraires sont relativement élaborées. En effet, certains corps sont individuellement recouverts de plâtre puis d'une enveloppe textile (Castex et al., 2009: 290).

Des paillettes d'ambre, des fils d'or et différentes résines ont également été utilisées (fig. 2). L'organisation de ces tombes multiples et l'appareil funéraire dévolu à certains individus diffèrent nettement de tout ce qui est connu pour cette catacombe.

1. Réalisée à l'Université de Bordeaux 3 et 1 , sous la direction respective de R. Vergnieux et D. Castex.
En effet, cette dernière est classiquement organisée en couloirs rectilignes au sein desquelles les individus sont inhumés soit dans des niches individuelles creusées dans la paroi (loculi), surmontées ou non d'un arc décoratif (arcosalia) ou encore en chambres funéraires regroupant plusieurs sépultures individuelles par famille ou par corporation (cubicula). Les sépultures du secteur central représentent un ensemble de salles en étoile au sein desquelles ont été mis au jour un nombre important d'individus de 74 à plus de 150 à l'heure actuelle et un nombre total d'individus estimés à plus de 3000 pour seulement 7 salles (Castex et al., 2009, 2011). Hors ces nouvelles tombes multiples du secteur central, il a pu être estimé que la population inhumée au sein de la catacombe représente entre 20000 et 25000 individus (Guyon, 1987; Guyon, 2004).

L'utilisation du secteur central en tant qu'espace sépulcral date de la fin du $\mathrm{I}^{\mathrm{er}}$ au milieu du $\mathrm{III}^{\mathrm{e}}$ siècle apr. J.-C. L'étude et la compréhension globale de ce secteur sont particulièrement difficiles en raison du nombre d'individus inhumés au sein d'une même tombe et des pratiques funéraires complexes dont certains ont fait l'objet. Les différentes cavités présentent une accumulation de squelettes en connexion, plus ou moins bien conservés et séparés par des passes de sédiments qui permettent d'identifier des niveaux de dépôts (fig. 3). Les différentes fouilles menées ont eu comme premier objectif de chercher à comprendre le fonctionnement de ces ensembles et à démontrer la simultanéité des dépôts, un des arguments permettant la caractérisation d'une sépulture multiple (Duday, 2007: 15-21). L'accumulation d'un grand nombre de vestiges osseux, estimé à plus de 3000 et les observations taphonomiques ont amené les acteurs de la fouille à émettre l'hypothèse de sépultures multiples en rapport avec une crise de mortalité, c'est-à-dire un épisode de mortalité anormale (Castex et al., 2011: 279).

Afin d'élaborer la méthodologie nécessaire à la modélisation tridimensionnelle, nous nous sommes focalisés sur deux tombes qui représentaient des ensembles clos, clairement individualisés des autres espaces funéraires.

Notre choix s'est donc porté sur les tombes 16 et 18 fouillées exhaustivement entre 2005 et 2006 et contenant respectivement 76 et 78 individus. La tombe 18 comporte 78 individus répartis sur 80 centimètres d'épaisseur et séparés par des passes de sédiments qui ont permis d'identifier 11 phases ou niveaux de dépôts successifs ${ }^{2}$.

Elle est de forme quadrangulaire (2,6 mètres nord-sud sur 1,6 mètre d'est en ouest) avec un plafond d'une hauteur de

2. Une passe de sédiment est une couche de terre ou unité stratigraphique qui vient sceller le dépôt d'un ensemble d'individus ce qui constitue un niveau de dépôt. 


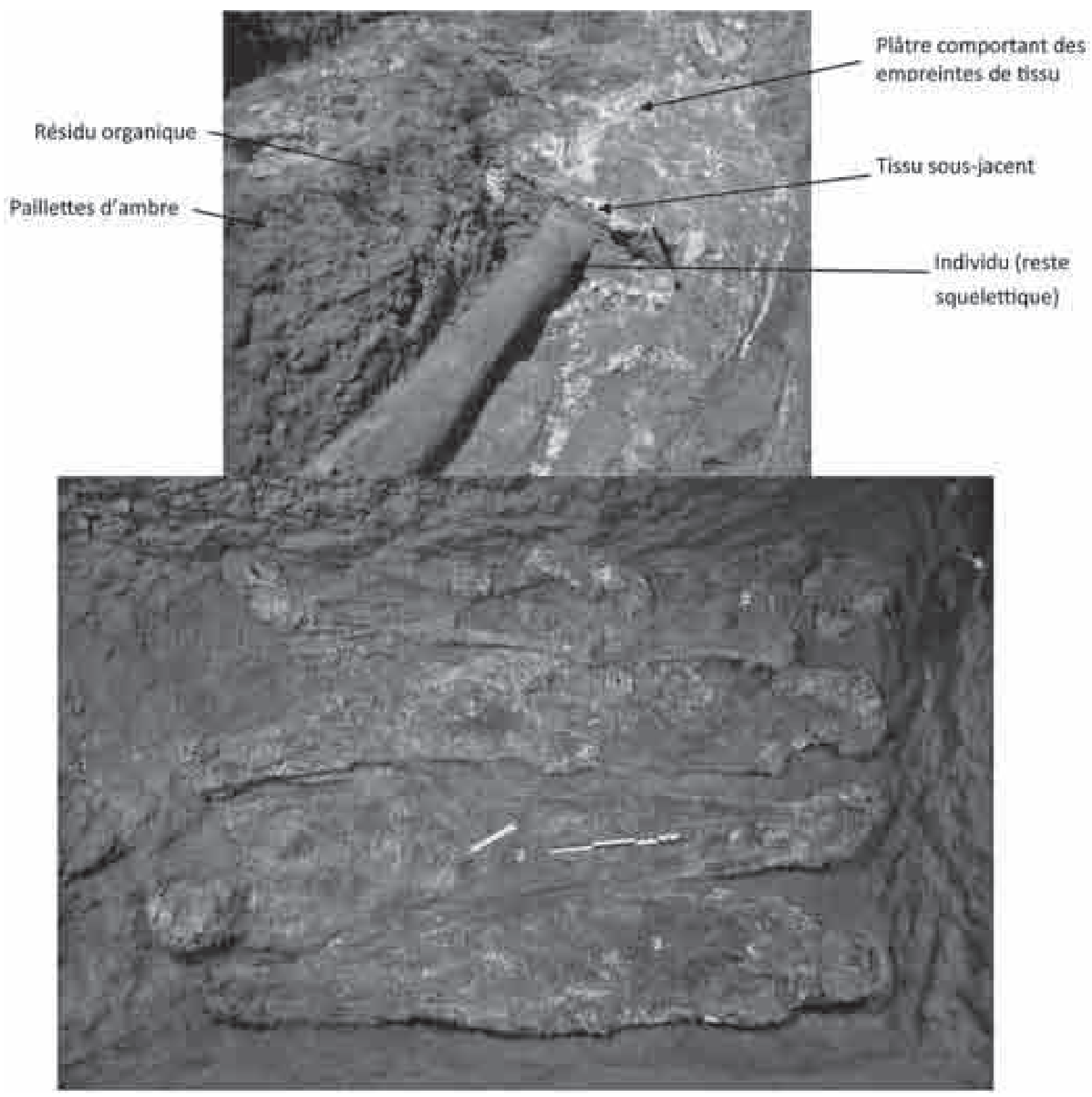

Figure 2: L’appareil funéraire, fémur pris dans une gangue de plâtre, T16 niveau 1 de dépôt (cl. mission 2005). Figure 2: An example of funerary practices, femur in a plaster matrix and the first individuals placed in tomb 16. 

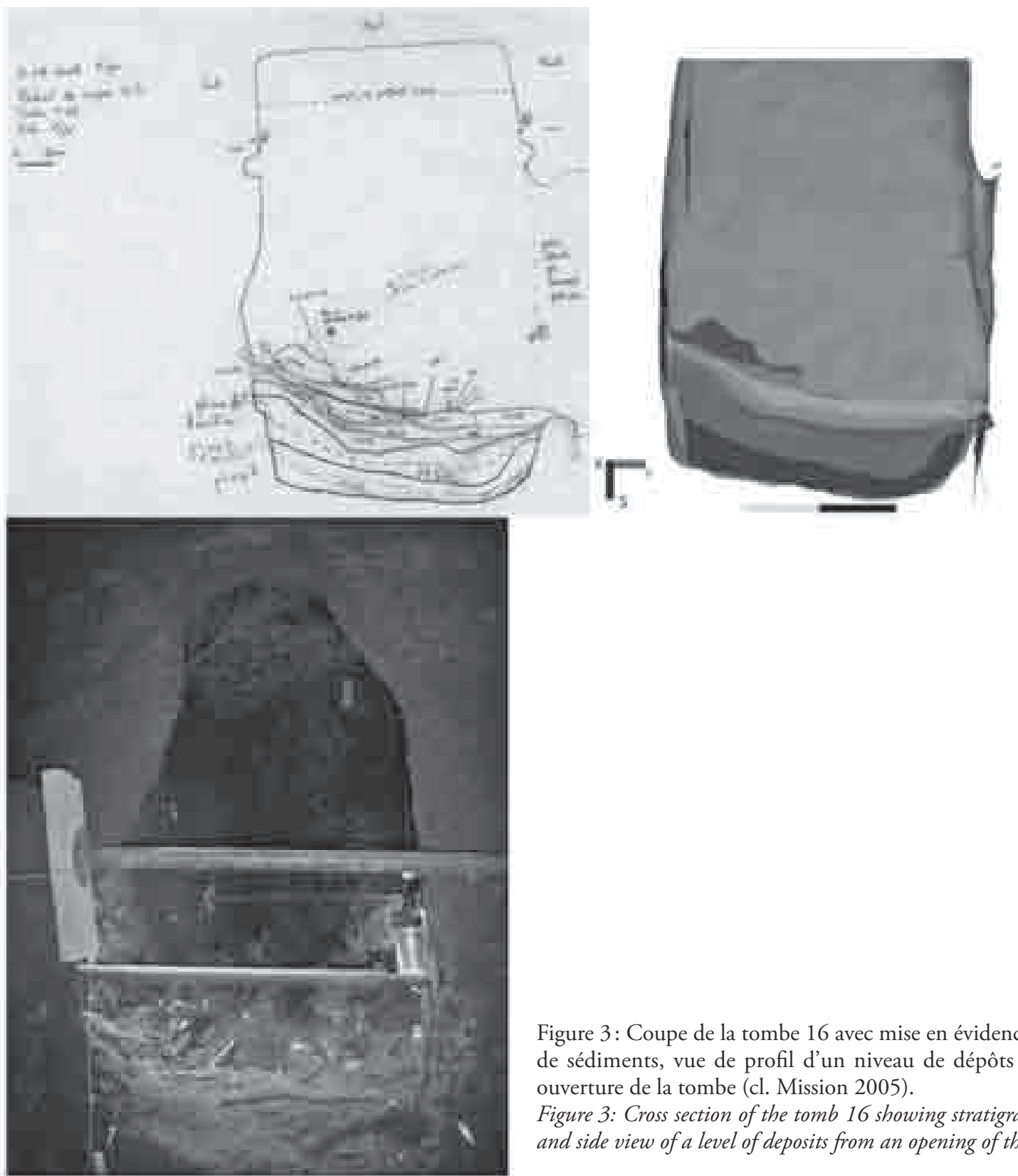

Figure 3: Coupe de la tombe 16 avec mise en évidence des passes de sédiments, vue de profil d'un niveau de dépôts depuis une ouverture de la tombe (cl. Mission 2005).

Figure 3: Cross section of the tomb 16 showing stratigraphical units and side view of a level of deposits from an opening of the tomb. 

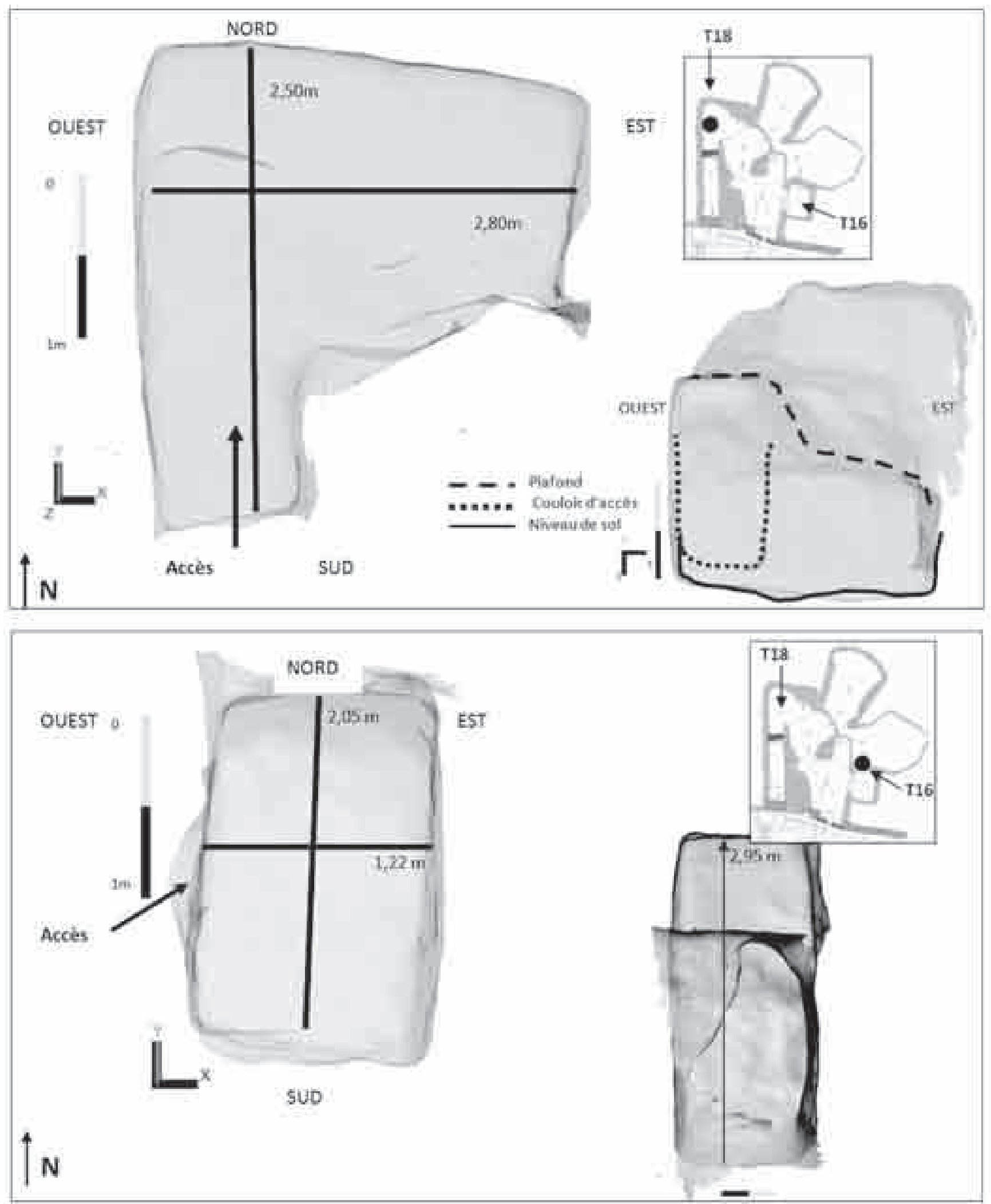

Figure 4: Tombe 18, vue supérieure et vue de la façade sud (en haut), Tombe 16 vue supérieure et vue de la façade sud (en bas). Figure 4: Top view of tomb 18 and north side view (top), top view of tomb 16 and north side view (bottom). 
2,10 mètres, un appendice au sud est identifié comme un couloir d'accès à la tombe.

La tombe 16 contient, quant à elle, 76 individus sur 70 centimètres et repartis en 9 niveaux de dépôts séparés par des passes de sédiment; elle est de forme rectangulaire (2,05 mètres du nord au sud et 1,20 mètres d'ouest en est), l'entrée est située sur la façade ouest de la tombe à une hauteur de 2 mètres (fig. 4). Afin de comprendre le fonctionnement de ces tombes, il était primordial d'appréhender la chronologie relative des différents dépôts et donc de recourir à une analyse taphonomique.

\section{Méthodes}

Selon les principes de l'Archéothanatologie, lors de l'étude des sépultures, le mort est placé au centre du discours mais cette discipline va bien au-delà de l'étude ostéologique et la compréhension de la logique des dépôts constitue un des axes fondamentaux et prend tout son sens dans le cadre des sépultures à dépôts multiples (Boulestin et Duday, 2005: 17-30). Il était donc indispensable de pouvoir appréhender le positionnement de chaque os par le biais des enregistrements de terrain afin de le restituer pour l'étude et ainsi comprendre le positionnement de l'individu et les gestes funéraires associés. En effet, pour appréhender la chronologie des dépôts et ainsi le fonctionnement de l'espace sépulcral, il a été nécessaire de pouvoir restituer l'image originelle de la tombe. Les visualisations proposées jusqu’à présent sont fondées sur des documents bidimensionnels (relevés, dessins, photographies) faisant ainsi appel à la capacité de représentation mentale des chercheurs pour appréhender le positionnement de l'individu (les contiguïtés articulaires, les dislocations) et les relations entre les individus, telles que les superpositions et les imbrications ${ }^{3}$, à l'aide des seules altitudes. Dans le cas des sépultures multiples notamment celles de la catacombe des Saints Pierreet-Marcellin, la superposition des calques ne permettait pas la lecture directe des informations au vu du nombre très important d'individus concernés.

En effet, sur ce type de support bidimensionnel, il est particulièrement difficile d'appréhender le positionnement relatif des individus entre eux puisqu'il est nécessaire de recalculer systématiquement les altitudes.

3. L'imbrication peut concerner un ou plusieurs individus et se caractérise par un entremêlement des corps avec le passage dessus et dessous d'au moins une partie du corps. Dans la plupart des cas, les connexions anatomiques sont conservées et une mise en équilibre des ossements peut être observée, preuve d'une décomposition conjointe et donc d'un dépôt simultané.
De plus, les représentations sont souvent simplifiées pour des questions de temps consacré à l'enregistrement notamment dans le cadre de fouille préventive, les segments osseux $^{4}$ ne sont donc pas toujours aisément identifiables lorsqu'ils sont schématisés sous la forme de bâtons. De même, lorsque la représentation est anthropomorphe, les relations entre les différents individus ne sont pas toujours explicites (fig. 5). La stratégie d'intervention et de recueil des données est tributaire du temps imparti et surtout de la complexité de ces ensembles, ces deux techniques de représentation ont été utilisées pour les sépultures multiples d'Issoudun ${ }^{5}$ et ont permis par leur précision de fournir les éléments indispensables à toute étude, toutefois l'émergence de nouveaux outils a permis de traiter différemment les informations similaires recueillies au sein du secteur central de la catacombe des Saints Pierre-et-Marcellin.

La méthode mise au point se propose de faciliter ce travail de restitution mentale des ensembles funéraires en produisant une scène numérique fondée sur la documentation acquise sur le terrain et permettant de visualiser en trois dimensions l'ensemble des squelettes d'une même sépulture dans leur situation et environnement de découverte.

Pour la restitution des ossements et donc des individus, la méthodologie mise en place se fonde entièrement sur la documentation de terrain existante (Sachau et al., 2010 : 161-170, Sachau et al., 2011:251-222). Les relevés constituent la plus importante source d'informations puisqu'ils permettent de reproduire le positionnement des ossements en fonction des points d'altitudes relevés.

Ils nous permettent d'acquérir les coordonnées tridimensionnelles des ossements, c'est-à-dire le positionnement spatial en $\mathrm{X}, \mathrm{Y}$ et $\mathrm{Z}$ de chaque os relevé indispensable à son positionnement dans un référentiel 3D.

Les relevés bidimensionnels ${ }^{6}$ permettent, une fois numérisés et grâce à un logiciel de dessin vectoriel, d'acquérir un jeu de coordonnées pour chaque point reporté sur le plan d'après un point d'origine défini. Pour l'intégration des vestiges osseux au sein du modèle $3 \mathrm{D}$, l'altitude a été relevée pour chaque point reporté sur le calque et calculée ensuite en fonction de l'altitude de référence. Il a été nécessaire d'accorder un soin tout particulier à la prise des mesures et à l'erreur que pouvait engendrer la constitution d'un jeu de coordonnées a posteriori (fig. 6).

4. L'expression segment osseux est utilisée ici dans le sens d'un ensemble d'os formant un segment anatomique.

5. Indre, mission réalisée sous la direction de Ph. Blanchard, I. SouquetLeroy et D. Castex entre le 21/05/2002 et le 6/09/2002 sur un ensemble funéraire datée du XI ${ }^{\mathrm{e}}$-XIII ${ }^{\mathrm{e}}$ comprenant 14 fosses à inhumations multiples.

6. Réalisés sur des calques au $1 / 10^{\mathrm{e}}$ superposés à un papier millimétré, les mesures sont réalisées à partir d'un carroyage. 

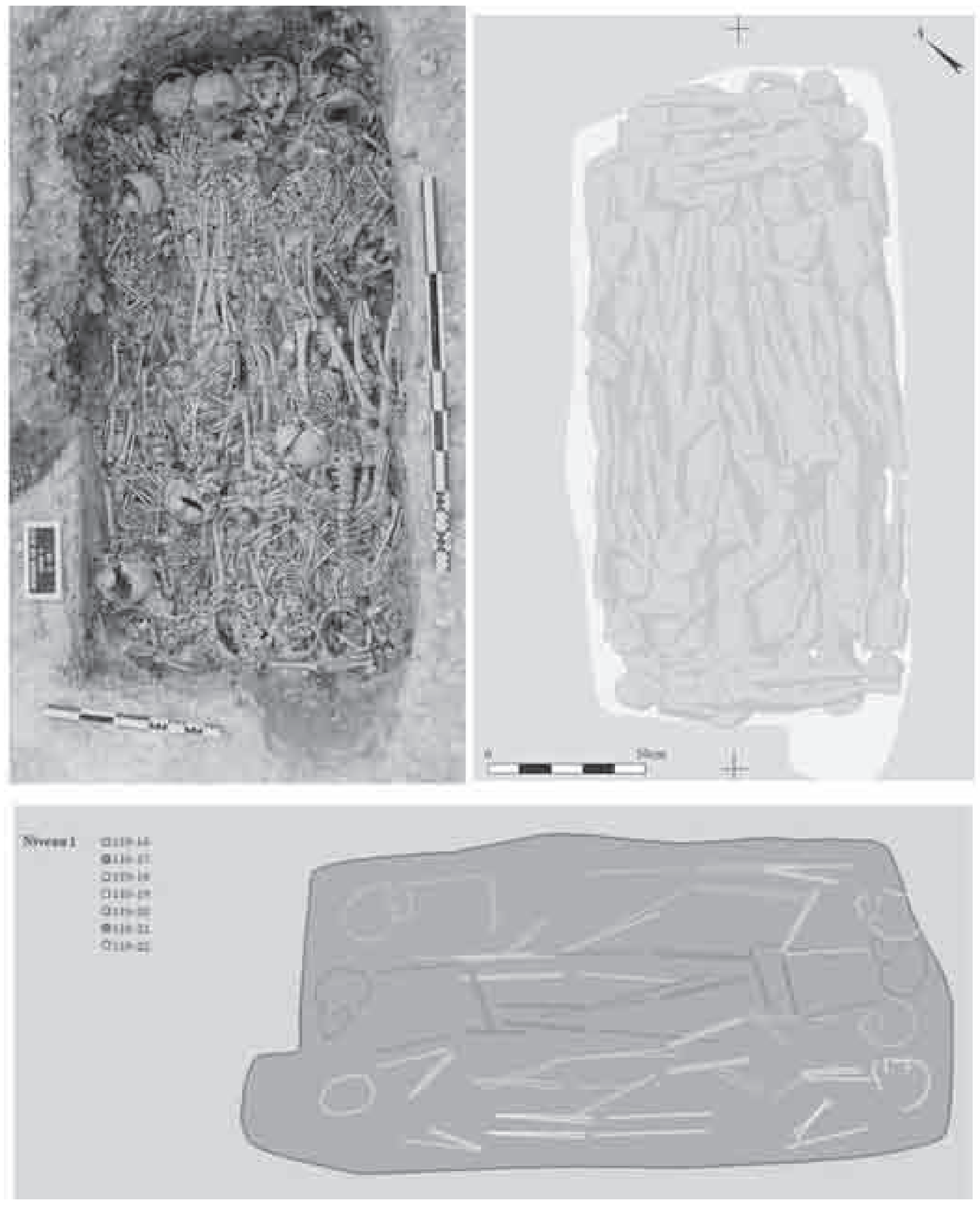

Figure 5: Exemple de représentation d'une sépulture multiple d'Issoudun (119) (Blanchard et al., 2011, vol. 2).

Figure 5: An example of several representation of a mass grave at Issoudun (number 119) (Blanchard et al., 2011, vol.2). 


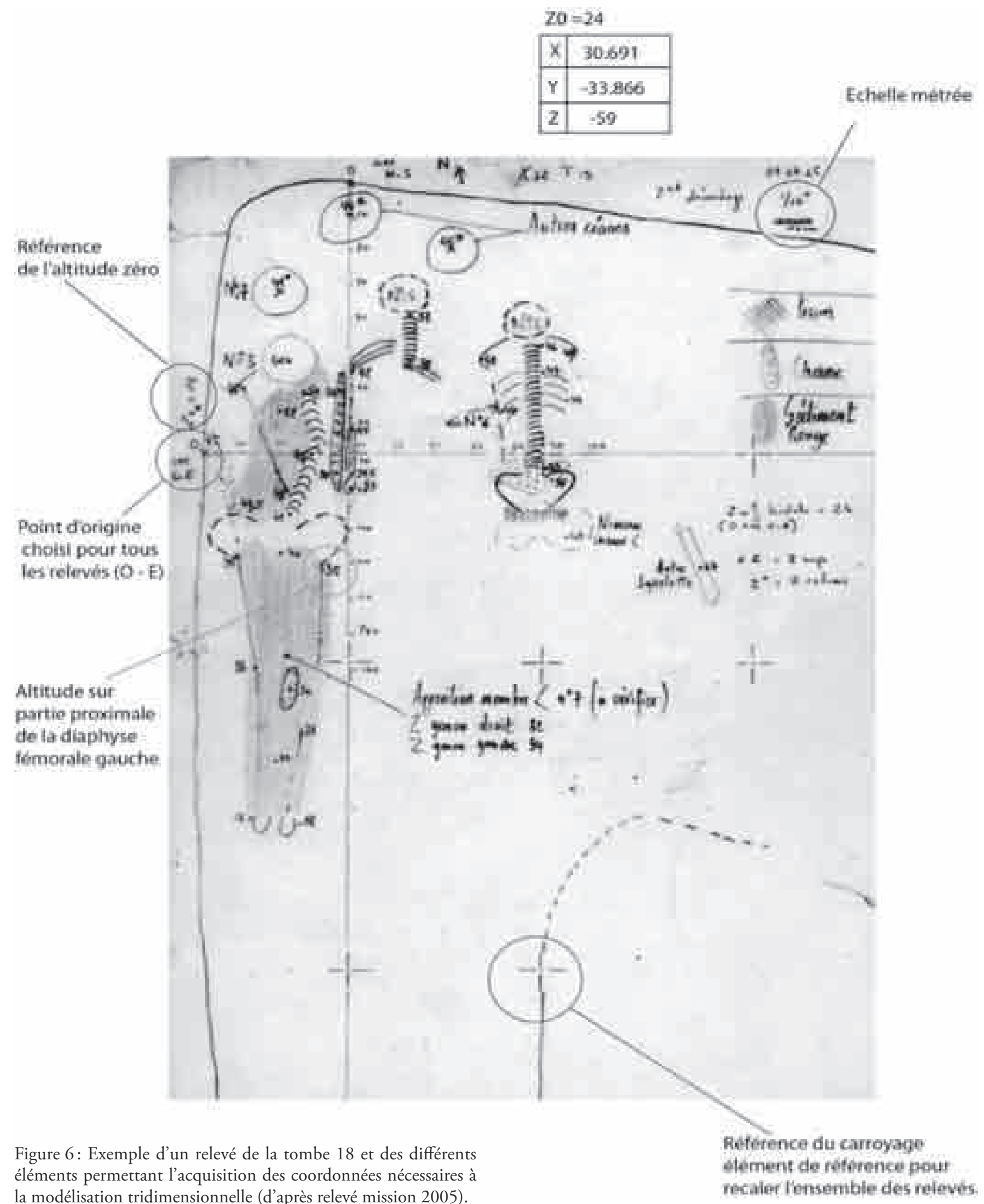

Figure 6: Cross section of the tomb 18. Figuration of elements used to acquire of $3 D$ coordinates (mission 2005). 

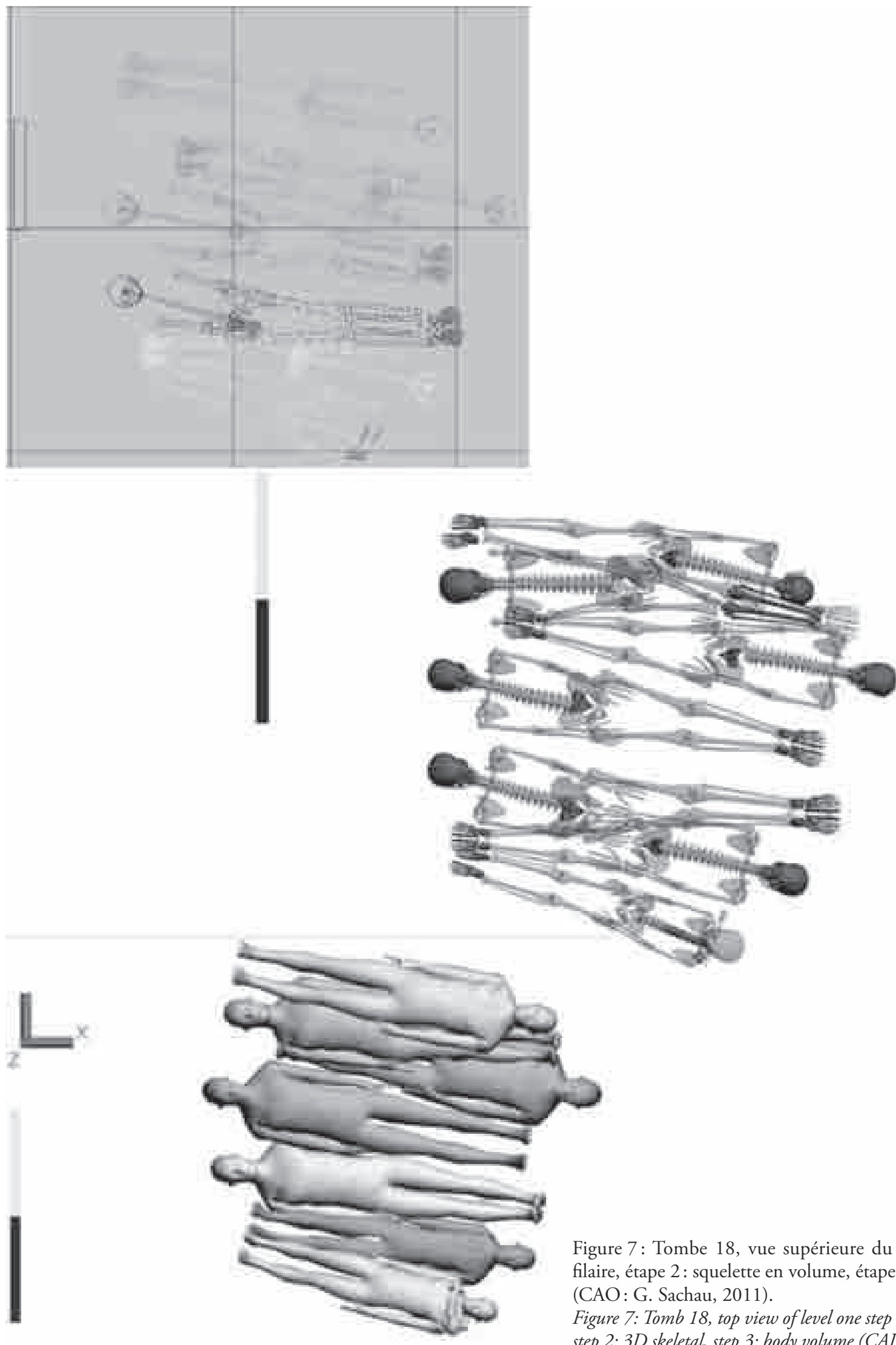

Figure 7: Tombe 18, vue supérieure du niveau 1, étape 1: filaire, étape 2: squelette en volume, étape 3: corps en volume (CAO: G. Sachau, 2011).

Figure 7: Tomb 18, top view of level one step 1: wireframe skeletal, step 2: 3D skeletal, step 3: body volume (CAD: G. Sachau, 2011). 
Les photographies et les minutes de terrain permettent quant à elles d'affiner la position de l'ossement, grâce à la visualisation des faces d'apparition et d'appréhender les vestiges associés, notamment les éléments de l'appareil funéraire. Une fois la documentation synthétisée par individu sous un tableur, la scène $3 \mathrm{D}$ a pu être créée.

Les individus ont été représentés sous trois formes: squelette filaire, squelette en volume, corps en volume. La représentation filaire du squelette de l'individu a été établie d'après les coordonnées acquises sur les relevés et les ossements de l'individu sont représentés par leur contour établi sur la base des fiches de démontages de terrain telles que celles établies par P. Courtaud ${ }^{7}$ (Courtaud, 1996: 161). Cette première étape de modélisation permet de vérifier les coordonnées relevées et l'ensemble des données recueillies pour chaque individu.

La deuxième étape a consisté en la mise en volume des ossements avec l'utilisation d'un modèle tridimensionnel de squelettes ${ }^{8}$. La modélisation des individus sous leur forme squelettique permet également d'identifier les parties conservées ou non par des jeux d'opacité et de transparence mettant ainsi en évidence les ossements présents dont la position est attestée, et ceux ayant été restitués. Les conditions de conservation n'ont pas permis de relever l'intégralité des ossements, toutefois, pour certains d'entre eux, la présence d'un négatif a permis de renseigner leur position.

Enfin, la troisième et dernière étape a permis de restituer les corps en volume des sujets déposés et ainsi appréhender le remplissage de la tombe, la gestion des corps par niveaux mais également par tombe (fig. 7). Il a été également possible, grâce à la reconstitution en volume au sein du modèle numérique scientifique, de visualiser les données sur le sexe des individus déposés ainsi que leur âge par la mise en place d'un code colorimétrique selon le genre et le degré de maturation. En effet, au sein de ces tombes multiples, il est intéressant de pouvoir mettre en évidence les différentes catégories d'âge pour les sujets immatures ou le sexe des individus inhumés afin d'en étudier le positionnement, la répartition et ainsi essayer de dégager des logiques de dépôts liés à l'un ou l'autre de ces critères.

Les deux tombes étudiées ont été restituées à l'aide de la photogrammétrie ${ }^{9}$ comme l'ensemble du secteur central de la catacombe des Saints Pierre-et-Marcellin. La restitu-

7. Ingénieur de recherche, UMR 5199, PACEA-A3P.

8. Le squelette employé fait partie des modèles libres de droit, en effet, lors de nos recherches il est apparu que les modèles 3D de squelette existaient dans le domaine scientifique notamment médical mais que l'échantillon utilisé pour leurs créations n'était pas connu; la validité de tels modèles ne pouvaient donc pas être évaluée.

9. Réalisée à l'aide de Pascal Mora (responsable Archéotransfert, université de Bordeaux 3). tion de l'espace funéraire constitue un élément important de l'étude: elle permet de visualiser les interactions entre les individus et la tombe (sol, parois, plafond) notamment les contraintes liées aux dimensions de la tombe ou encore à la morphologie des parois. Les caractéristiques de la catacombe (humidité, éclairage...) ne permettaient pas l'acquisition de l'espace funéraire à l'aide d'autres matériels d'acquisition 3D tel que le laserscan par exemple.

\section{ÉTUDE}

L'étude des deux tombes multiples a été facilitée par la représentation dans les scènes numériques tridimensionnelles de l'ensemble de la tombe (espace funéraire et individus déposés).

En effet, la visualisation de la tombe ne s'appuie plus sur une reconstitution mentale individuelle mais sur un modèle tridimensionnel de l'état lors de la découverte, interactif, évolutif, et visualisable par tous. L'objectif a été d'établir une chronologie relative pour chacune des tombes 16 et 18 qui pourra par la suite être confrontée à l'ensemble des tombes du secteur central. Ces différents éléments permettront également de discuter de la simultanéité des dépôts et donc de caractériser ces espaces funéraires particuliers.

La chronologie interne propre à chacune des tombes est appréhendée grâce à l'étude des interrelations osseuses - c'est-à-dire l'étude de l'imbrication, de la superposition et de la juxtaposition des individus - mais également grâce la compréhension de la gestion des corps, que l'analyse soit réalisée sur les individus sous la forme de squelette ou sous forme de corps en volume.

Les différents niveaux d'analyse nous ont permis de déterminer qu'il existait une simultanéité au sein des niveaux de dépôts mais également entre les niveaux de dépôts grâce à l'imbrication des corps mais également à l'observation de la déformation des corps liée aux poids des niveaux sus ou sousjacents entre autres (Duday, 2007 : 15-21). Nous ne détaillerons pas dans cet article tous les arguments développés qui nous permettent d'aboutir à cette conclusion mais seulement les éléments qui n'auraient pas pu être identifiés sans l'utilisation d'une scène numérique tridimensionnelle.

Un élément important a été la possibilité de visualiser les relations entre les individus et notamment les imbrications et ce dans chacune des deux tombes étudiées. Les imbrications constituent une des preuves des dépôts simultanés et la mauvaise conservation des vestiges osseux n'a pas permis de toutes les observer lors de la fouille. L'étude des relations entre les individus à partir du modèle numérique 3D nous a donc permis de compléter les observations de terrain en 
ajoutant de nouveaux cas d'imbrication et ainsi renforcer les arguments en faveur d'un dépôt simultané de plusieurs individus au sein des tombes 16 et 18 . Ces éléments sont autant d'indices alimentant la discussion autour d'une crise de mortalité.

Les espaces libres interprétés comme des espaces de circulation ont été plus facilement identifiés grâce à la superposition des différents niveaux de dépôts ${ }^{10}$ et des passes de sédiments dont l'épaisseur varie en fonction des vestiges sous-jacents, c'est-à-dire s'il y a ou non un individu à recouvrir. La visualisation conjointe des passes de sédiments restitués et des individus modélisés a permis d'étayer ces observations.

L'étude de la tombe 18 nous a permis de mettre en évidence un ensemble d'espaces de circulation utilisés puis comblés selon les niveaux de dépôt par un ou plusieurs individus. La restitution des différents niveaux de dépôts au sein de la tombe 18 nous a permis d'identifier à l'ouest un espace de circulation dans le prolongement du couloir et surélevé par rapport au reste de la tombe d'une dizaine de centimètres. Il est utilisé pour venir déposer le premier niveau puis comblé lors du dépôt du deuxième niveau et réutilisé en partie ou complètement pour le dépôt des niveaux suivants.

La surface disponible est optimisée pour accueillir un maximum d'individus mais ces espaces liés à la circulation sont préservés après chaque apport de corps, tout comme l'horizontalité des différents niveaux de dépôts est maintenue par les passes de sédiment. Ces éléments démontrent que pendant toute la durée d'utilisation de la tombe, la localisation des corps est connue ce qui suggère qu'il y a eu transmission de l'information.

Des observations similaires ont pu être faites au sein de la tombe 16 où au cours du dépôt d'un même niveau, un espace est laissé libre le long des parois ouest (où se situe l'entrée) et sud pour permettre le dépôt des corps dans la largeur avant d'être finalement comblé par le dépôt d'un ou plusieurs individus. L'analyse des espaces libres, interprétés comme des espaces de circulation, nous ont amené à penser qu'il existait une simultanéité entre les niveaux de dépôts bien qu'ils soient séparés par des passes de sédiments.

La mise en volume des individus au sein des tombes nous a permis de discuter plus précisément les rythmes des dépôts et ainsi appréhender une chronologie d'utilisation de la tombe à partir de la logique globale de remplissage de la tombe et n'ont plus des chronologies relatives au sein des niveaux.

10. Individualisés d'après les passes de sédiments observés lors de la fouille, 11 au sein de la tombe 18 et 9 au sein de la tombe 16 .
Ainsi, nous avons pu observer que les individus composant les onze niveaux de dépôts de la tombe 18 n'ont pu être déposés en un seul et même temps car la hauteur cumulée des corps dépasse celle du plafond de la salle funéraire.

Seuls cinq niveaux consécutifs auraient pu être déposés dans des laps de temps très court; à l'inverse nous avons ainsi pu mettre en évidence que le dépôt des quatre derniers niveaux nécessitait l'entière décomposition des sept premiers niveaux de dépôts. Les dépôts ont donc dû être interrompus pendant un laps de temps qu'il n'est pas possible d'appréhender en termes d'années ou de mois, mais qui constitue tout de même un jalon chronologique.

Il en est de même pour la tombe 16, les 9 niveaux complets n'ont pas pu être déposés en même temps, le dépôt maximal possible étant de 5 niveaux si l'ensemble des corps n'était pas décomposé (fig. 8).

Bien entendu, il ne s'agit que de calcul de maxima et il est tout à fait envisageable que la population inhumante ait attendu la décomposition de chaque niveau avant de procéder au dépôt de nouveaux corps mais la présence d'importants effets de cuvette, c'est-à-dire la déformation des corps sous le poids des niveaux sus-jacents nous laisse penser qu'il y a bien eu plusieurs niveaux successifs déposés dans un laps de temps relativement court entraînant ainsi la déformation des corps lors de leur décomposition. En effet, les corps soumis à un poids lors de la décomposition adoptent la forme des niveaux précédents ou celui du profil de la tombe, l'effet de cuvette ainsi crée se matérialise par une altitude supérieure du crâne et des pieds par rapport au bassin.

Toutefois, l'ensemble des jalons chronologiques nous renseignent sur la durée relative de la crise de surmortalité et sur l'utilisation de l'ensemble des tombes. Ces différentes observations ont permis de confirmer les présupposés archéologiques formulés lors de la fouille mais qui n’auraient pu être démontrés sans la possibilité de simuler les volumes corporels.

L'utilisation d'une scène numérique tridimensionnelle a également simplifié la visualisation des caractéristiques de l'ensemble des individus selon le sexe ou encore selon le degré de maturation et ce quel que soit leur positionnement au sein de la tombe puisque tous les angles d'observations sont possibles.

L'étude de la répartition des individus a ainsi permis d'identifier que les individus de sexe féminin sont généralement utilisés pour combler des espaces libres de taille réduite, entre un individu et la paroi, dans une anfractuosité ou sur un ressaut de la paroi par exemple. Le format réduit des sujets immatures est utilisé de la même manière et ceux-ci sont positionnés entre des individus adultes ou 


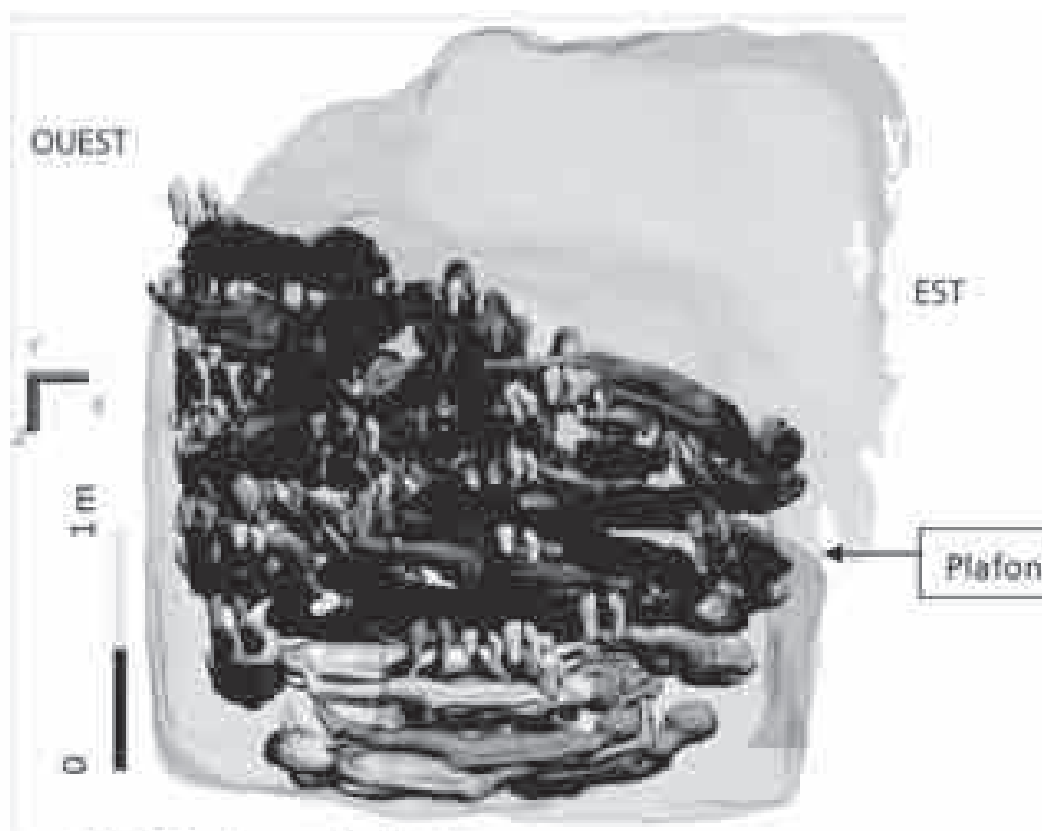

T18, 11 niveauxde dépóts

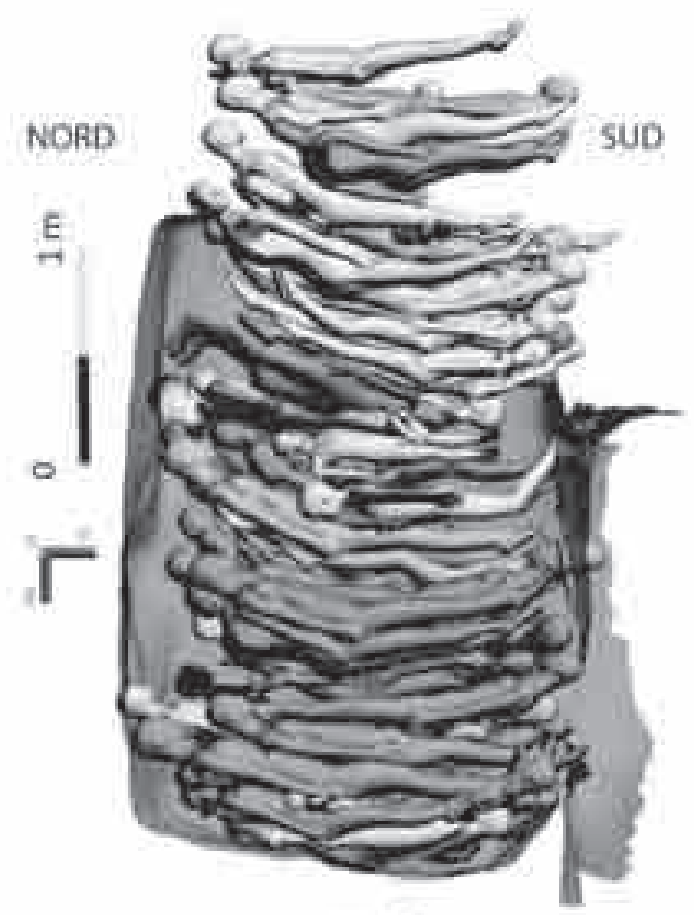

T16, 9 niveauxde dépôts

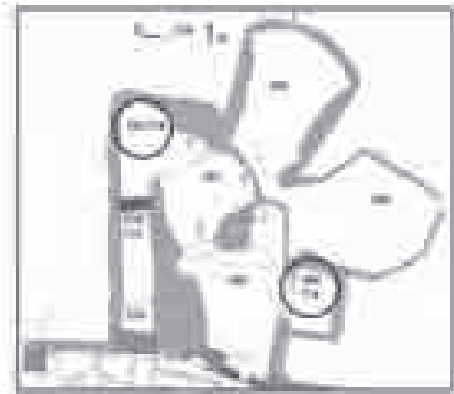

T18, 5 niveauxde depòts

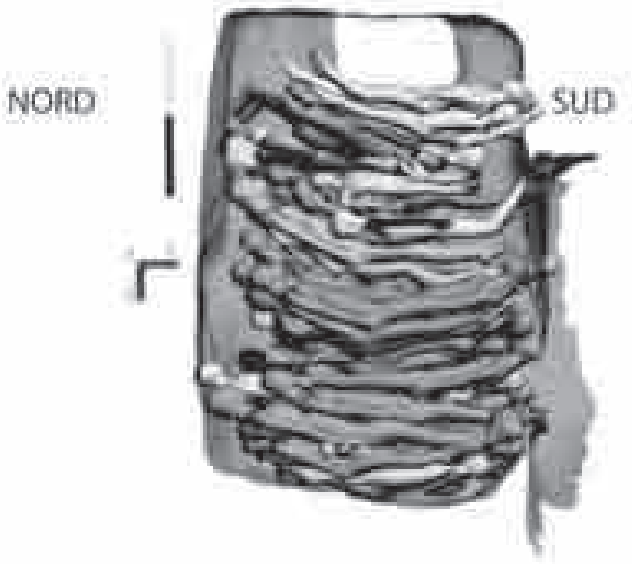

T16,5 niveaux de dépôts

Figure 8: Restitution de l'ensemble des niveaux de dépôts (volume corporel) et des niveaux maximaux atteints, T18 (en haut) et T16 (en bas) (CAO: G. Sachau, 2011).

Figure 8: Restitution of all level deposits with bodies' volume in each grave and maximal level possible to deposit individuals T18 (top) and T16 (bottom) (CAD: G. Sachau, 2011). 
contre les parois là ou un individu de format adulte n'aurait pu être déposé. L'étude de la répartition des individus selon leur caractéristique biologique permet ainsi d'appréhender toute la réflexion qui a précédé le dépôt des corps et les choix opérés en amont.

Ainsi, il apparaît clairement que pour les deux tombes étudiées il existe une gestion des corps, une sélection en surface ou lors du dépôt au sein de la tombe des sujets qui pourraient être en relation avec la préparation des corps. Cette gestion est particulièrement visible sur la tombe 18 ou le dépôt du premier niveau tient compte du format des individus et s'adapte ainsi à la morphologie du sol du fond de la tombe.

L'utilisation de l'espace funéraire modélisé revêt un caractère particulièrement intéressant pour la tombe 16 car un ensemble de prélèvements physico-chimiques (pour détecter la présence de résidus de liquides de putréfaction) a été réalisé sur une des parois. La confrontation des résultats avec la modélisation des corps en volume devrait se révéler riche d'informations puisque nous pourrons ainsi comparer les volumes de corps déposés théoriques avec les traces de décomposition. Ainsi nous pourrions mettre en évidence les différentes hauteurs atteintes par les corps au moment de la décomposition.

\section{APPORTS}

Au-delà de la simplification de l'échange entre les différents acteurs de la fouille, archéologues et anthropologues, la modélisation tridimensionnelle permet d'amener de nouveaux axes de réflexion grâce à la visualisation de l'ensemble des interactions dans un univers tridimensionnel. La modélisation tridimensionnelle propose une infinité de points de vue, du plus général au détail. De plus, la possibilité de générer des illustrations, des vidéos, simulant la succession des dépôts contribuent à faciliter les échanges entre chercheurs. Il s'agit d'une autre manière d'exporter le modèle scientifique mais la validité du travail en amont est la même puisque l'ensemble des données modélisées se fonde sur les données de fouille.

Par ailleurs, la création d'un modèle numérique contribue à la pérennisation des données car son élaboration nécessite la synthèse de l'intégralité de la documentation recueillie lors de la fouille (Vergnieux, 2008: 241-243; Vergnieux, 2010: 181-184). La relecture attentive de ces documents permet éventuellement de les corriger et de les enrichir des dernières observations réalisées lors des différentes campagnes de postfouille. La sauvegarde et la lecture des scènes numériques tridimensionnelles est tributaire du format du fichier, nous ne sommes pas sûrs que le logiciel existera encore dans le futur mais le recueil des coordonnées complètes de chaque os de chaque individu assure la reproductibilité de la scène sans être attachée à une version de logiciel.

\section{Conclusion}

Le modèle scientifique 3D nécessite un temps de conception plus long que la mise au propre des relevés de terrain mais s'appuie sur les mêmes données. Les techniques d'enregistrement actuel n'ont donc pas besoin d'être entièrement remises en cause mais pourront être améliorées grâce à l'échange entre le modeleur et l'archéologue avant le début des fouilles, mais également avec le développement de nouveaux outils, par une saisie immédiate des coordonnées et des informations principales. Le recours à un modèle 3D simplifie le travail de représentation mentale et par conséquence les échanges entre chercheurs, et il permet ainsi d'établir plus rapidement et plus précisément des comparaisons avec d'autres sépultures multiples, que ce soit en terme de gestion des corps ou d'appareil funéraire. L'accès à la diffusion quasi immédiate d'images ou de vidéos en fait un outil plus rapide sur le long terme et plus efficace.

La restitution tridimensionnelle permet également d'accéder à des états disparus et de pérenniser les informations en palliant au caractère destructeur de la fouille ce qui permettra également de revenir sur des travaux plus anciens concernant des sépultures multiples mais également d'utiliser ces nouvelles techniques dans le cas des sépultures collectives.

\section{Bibliographie}

Blanchard Ph., Castex D., Souquet-Leroy I., Poulle P., 2003. Issoudun, centre de l'image et du multimédia. Archéopages, mars 2003, 9: 40-41.

Blanchard Ph., Souquet-Leroy I., Castex D., 2011. Issoudun, Indre, Les Champs Elysées, (Centre de l'image), Témoignages de deux crises de mortalité moderne dans le grand cimetière, volume 1 : texte et figure, volume 2 : catalogue des sépultures individuelles, volume 3 : catalogue et étude pathologique des sépultures multiples, volume 4 : annexes: études documentaire et céramique, inventaires techniques, Inrap, 2011, rapport de fouille, inédit.

Boulestin B., Duday H., 2005. Ethnologie et archéologie de la mort: de l'illusion des références à l'emploi d'un vocabulaire. In Mordant C. et Depierre G. (dir.), Les pratiques funéraires à l'âge du bronze en France. Actes de la table ronde de Sensen-Bourgogne, (10-12 juin 1998), Paris/Sens, Éd. du CTHS/ Société archéologique de Sens: 17-30. 
Castex D., Blanchard Ph., Giuliani R., Ricciardi M., 2007. Les ensembles funéraires du secteur central de la catacombe des Saints Pierre-et-Marcellin (Rome, $\mathrm{I}^{\mathrm{er}}-\mathrm{III}^{\mathrm{e}}$ s.) : caractérisation, hypothèses d'interprétations et perspectives de recherches. Mélanges de l'École Française de Rome. Antiquité, 119-1: 274 282.

Castex D., Cartron I. (dir.), 2005. Épidémies et crises de mortalité du passé, Actes des séminaires (année 2005) de la Maison des Sciences de l'Homme, Ausonius éd., Bordeaux.

Courtaud P., 1996, "Anthropologie de sauvetages ": vers une optimisation des méthodes d'enregistrement, présentation d'une fiche anthropologique. Bulletin et Mémoires de la Société d'Anthropologie de Paris, t. 8, (3-4): 157-167.

DudAY H., 2007. Les preuves archéologiques d'une crise brutale de mortalité: simultanéité du dépôt des cadavres, simultanéité des décès? In Castex D, Cartron I, Épidémies et crises de mortalité du passé, Acte des séminaires (année 2005) de la Maison des Sciences de l'Homme, Ausonius éd., études 15: 15-21.

Giuliani R., Castex D., en coll. avec Blanchard Ph. et Coquerelle M., 2007. La scoperta di un nuovo santuario nella catacomba dei SS. Marcellino e Pietro e lo scavo antropologico degli insiemi funerari annessi. Risultati preliminari di un'indagine multidisciplinare. Rendiconti della Pontificia Accademia Romana di Archeologia, LXXIX 2006-2007: 83-124.

Sachau G., Castex D., Mora P., Vergnieux R., 2010. Modélisation de deux ensembles funéraires de la catacombe des Saints Pierre et Marcellin à Rome: objectifs et méthodes. In Vergnieux R., Delevoie C., Actes du colloque Virtual Retrospect 2009, Archéovision 4, Ausonius éd., Bordeaux: 161-170.

Sachau G., Castex D., 2011. Apport de la modélisation tridimensionnelle à l'analyse des sites de stratifications complexes à travers l'exemple des tombes multiples de la catacombe des Saints Pierre-et-Marcellin. In Père Chr., Rollier J., Actes du colloque Arch-I-Tech 2010, Archéovision 5, Ausonius éd., Bordeaux: $215-222$.

Vergnieux R., 2008. Archeogrid - Des modèles numériques 3D pour quoi faire? In Vergnieux R., Delevoie C. (éd.), Actes du colloque Virtual Retrospect 2007, Archéovision 3, Ausonius éd., Bordeaux: 241- 245.

Vergnieux R., 2010. Sauvegarder les données numériques 3D du patrimoine. In Vergnieux R., Delevoie C., Actes du colloque Virtual Retrospect 2009, Archéovision 4, Ausonius éd., Bordeaux: 181-184. 